\title{
Gastrosplenic fistula secondary to lymphoma, manifesting as upper gastrointestinal bleeding
}

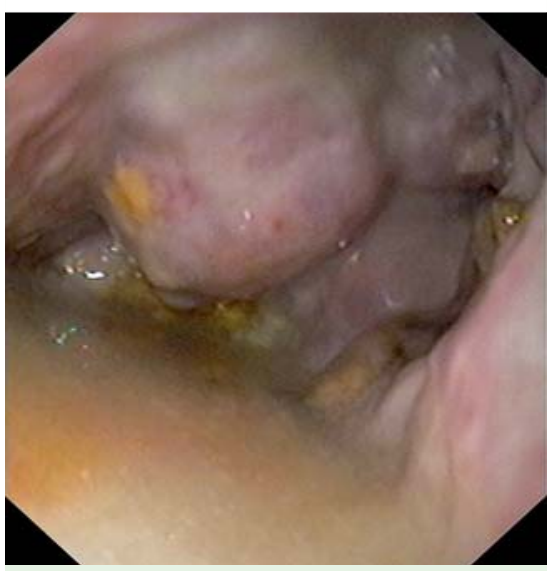

Fig. 1 Endoscopic view following insertion of an endoscope into the cavitary lesion via a fistula. The patient was 68 years old and presented with hematemesis.

Gastrosplenic fistula is a rare clinical condition that occurs mostly in association with splenic or gastric lymphomas [1]. We report a case of a gastrosplenic fistula that formed secondary to a diffuse large B-cell splenic lymphoma and presented as upper gastrointestinal bleeding with hematemesis.

A 68-year-old man was admitted to our hospital due to hematemesis. Endoscopy revealed a large cavitary lesion with necrotic material at the posterior gastric fundus ( Fig. 1), and histological examination of biopsy specimens taken from the cavity showed diffuse large B-cell nonHodgkin's lymphoma ( $\bullet$ Fig. 2). Computed tomography (CT) revealed a large splenic mass with a fistulous tract between the gastric lumen and an air-filled necrotic splenic cavity ( Fig. 3). Surgical treatment with en bloc resection was preferred as initial treatment rather than chemotherapy, to avoid the risk of recur- rence of bleeding. Chemotherapy was started 30 days postoperatively. The patient continues to remain under evaluation.

In cases of splenic lymphoma, close proximity of the gastric fundus to the spleen and the presence of the gastrosplenic ligament facilitate the union of these organs and the formation of a fistula as a result of growth and spread of the tumor cells, as well as tumor necrosis or infection. The gastrosplenic fistula may occur either spontaneously or secondary to chemo- or radiation therapy, with the possibility of massive hematemesis secondary to erosion of the splenic vessels by the gastric contents [2,3]. Endoscopy usually aids direct visualization of the fistulous tract opening, which is seen as a cavitary lesion, and abdominal CT provides the definite diagnosis. Although there are reports of gastrosplenic fistula resolving with chemotherapy, early radical surgical resection seems to be the best initial treatment approach to avoid re-bleeding. Gastrosplenic fistula should be suspected in patients with splenic lymphoma and upper gastrointestinal bleeding. Definitive surgical treatment is warranted in such cases to avoid massive gastrointestinal bleeding $[4,5]$.

Endoscopy_UCTN_Code_CCL_1AB_2AD_3AB

\section{Competing interests: None}

D. Dellaportas ${ }^{1}$, A. Vezakis ${ }^{1}$, G. Fragulidis $^{1}$, M. Tasoulis ${ }^{1}$, E. Karamitopoulou ${ }^{2}$, A. Polydorou ${ }^{1}$

1 2nd Department of Surgery, Aretaieion University Hospital, Athens, Greece

2 1st Department of Pathology, Aretaieion University Hospital, Athens, Greece

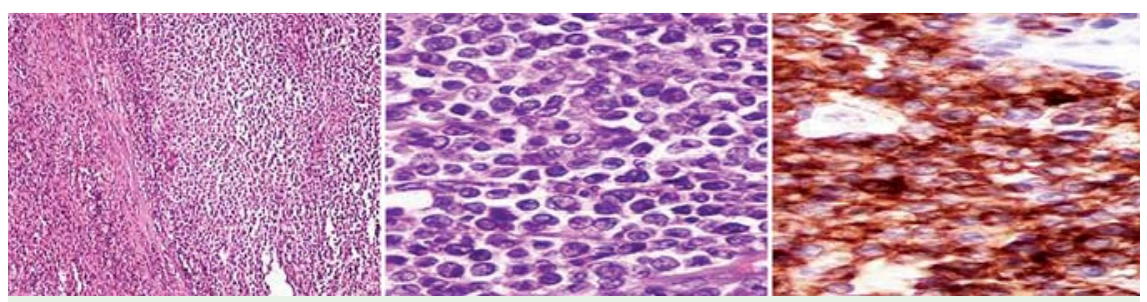

Fig. 2 a, b Microscopic view of infiltration of the splenic parenchyma by diffuse large B-cell lymphoma (a original magnification $\times 100$; b original magnification $\times 400$ ). $\mathbf{c}$ Immunostaining of the neoplastic population with CD79a.

\section{References}

1 Choi JE, Chung HJ, Lee HG. Spontaneous gastrosplenic fistula: a rare complication of splenic diffuse large cell lymphoma. Abdom Imaging 2002; 27: 728 - 730

2 Bird MA, Amjadi D, Behrns KE. Primary splenic lymphoma complicated by hematemesis and gastric erosion. South Med J 2008; 95: 941 - 942

3 Hiltunen KM, Mattila J, Helve O. Massively bleeding gastrosplenic fistula following cytostatic chemotherapy of a malignant lymphoma. J Clin Gastroenterol 1991; 13: 478-481

4 Kerem M, Sakrak O, Yilmaz TU et al. Spontaneous gastrosplenic fistula in primary gastric lymphoma: Surgical management. Asian J Surg 2006; 29: 287-290

5 Puppala S, Williams R, Harvey J et al. Spontaneous gastrosplenic fistula in primary gastric lymphoma: Case report and review of literature. Clin Radiol Extra 2005; 60: 20 22

\section{Bibliography}

DOI $10.1055 / \mathrm{s}-0030-1256935$

Endoscopy 2011; 43: E395

(c) Georg Thieme Verlag KG Stuttgart · New York . ISSN 0013-726X

\section{Corresponding author \\ D. Dellaportas}

2nd Department of Surgery

Aretaieion Hospital

University of Athens

76 Vassilissis Sofias Ave

11528 Athens

Greece

dellapdio@gmail.com

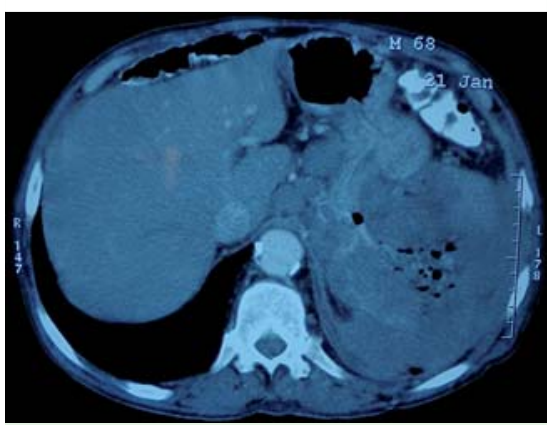

Fig. 3 Abdominal computed tomography (CT) scan showing a large splenic mass infiltrating the gastric fundus. The mass contained air bubbles, suggestive of communication with the stomach. 